Research Article

\title{
Neurodevelopmental Disorder Traits in Taijin-Kyofu-sho and Social Anxiety Disorder: A Cross-Sectional Study among University Students
}

\author{
Kosuke Kajitani $(\mathbb{D}$, Rikako Tsuchimoto, Yusaku Omodaka, Tomoko Matsushita, \\ Hideaki Fukumori, Takeshi Sato, and Jun Nagano \\ Center for Health Sciences and Counseling, Kyushu University, 744, Motooka, Nishi-ku, Fukuoka 819-0395, Japan \\ Correspondence should be addressed to Kosuke Kajitani; kkajitani@chc.kyushu-u.ac.jp
}

Received 2 May 2021; Revised 20 August 2021; Accepted 31 August 2021; Published 17 September 2021

Academic Editor: Joan Busner

Copyright (c) 2021 Kosuke Kajitani et al. This is an open access article distributed under the Creative Commons Attribution License, which permits unrestricted use, distribution, and reproduction in any medium, provided the original work is properly cited.

\begin{abstract}
Taijin-Kyofu-sho is an East Asian culture-bound anxiety disorder with similarities to social anxiety disorder. However, few studies have examined these two disorders from the perspective of neurodevelopmental disorders. This study is aimed at examining the association of Taijin-Kyofu-sho and social anxiety disorder with the attention-deficit/hyperactivity disorder (ADHD) trait and autism spectrum disorder (ASD) trait. The Liebowitz Social Anxiety, Taijin-Kyofu-sho, and Adult AttentionDeficit/Hyperactivity Disorder Self-Report scales and the 16-item Autism-Spectrum Quotient were administered to 818 university students. Participants were divided into four groups: control (neither Taijin-Kyofu-sho nor social anxiety disorder), pure Taijin-Kyofu-sho (Taijin-Kyofu-sho alone), pure social anxiety disorder (social anxiety disorder alone), and mixed TaijinKyofu-sho-social anxiety disorder (both Taijin-Kyofu-sho and social anxiety disorder). We used logistic regression analysis to examine whether the ADHD trait and ASD trait were associated with Taijin-Kyofu-sho and social anxiety disorder. ASD trait was significantly associated with pure Taijin-Kyofu-sho $(p=0.006$, odds ratio: 3.99). Female sex and ADHD trait were significantly associated with pure social anxiety disorder (sex: $p=0.013$, odds ratio: 2.61; ADHD trait: $p=0.012$, odds ratio: 2.46). Female sex, ADHD trait, and ASD trait were significantly associated with mixed Taijin-Kyofu-sho-social anxiety disorder (sex: $p=0.043$, odds ratio: 2.16; ADHD trait: $p=0.003$, odds ratio: 2.75; ASD trait: $p<0.001$, odds ratio: 16.93). Neurodevelopmental disorder traits differed between individuals with Taijin-Kyofu-sho and those with social anxiety disorder. Overall, our study reveals that Japanese individuals with the ADHD or ASD traits are at a heightened risk of developing Taijin-Kyofu-sho or social anxiety disorder.
\end{abstract}

\section{Introduction}

Neurodevelopmental disorders (NDDs) result from impairments in brain growth and development that lead to emotional and behavioral problems that begin in early childhood and persist into adulthood. According to the Diagnostic and Statistical Manual of Mental Disorders, Fifth Edition (DSM-5), NDDs are characterized by developmental deficits of varying severity in personal, academic, or occupational functioning [1]. Among NDDs, autism spectrum disorder (ASD) and attention-deficit/hyperactivity disorder (ADHD) often cause significant problems for college students. Students with ADHD or ASD are at risk of poor academic achievement and social isolation and are less likely to pursue a college education [2-4]. Furthermore, in young adults, ADHD or ASD is often comorbid with other psychiatric disorders, including anxiety disorder, mood disorder, and obsessive-compulsive disorder [5-7], which worsens their social adaptation [8]. In fact, having a mental health comorbidity is a negative predictor of quality of life for adults with ASD [9], and complications of psychiatric disorders are associated with poor academic performance in children with 
ADHD [10]. Thus, it is important to consider how comorbid disorders affect the quality of life of students with NDDs.

Social anxiety disorder (SAD) is an anxiety disorder characterized by marked fear of social situations in which the individual is exposed to possible scrutiny by others [11]. Examples of social situations include social interaction (e.g., having a conversation and meeting unfamiliar people), being observed (e.g., eating or drinking), and performing in front of others (e.g., giving a speech). Accumulating evidence suggests that patients with NDDs often have SAD symptoms. For example, Freeth et al. examined the association between ASD and SAD using the Autism-Spectrum Quotient (AQ) and Liebowitz Social Anxiety Scale (LSAS) and indicated that British students with multiple autistic traits were more likely to report increased social anxiety than those with few autistic traits [12]. A systematic review by Spain et al. on the relationship between ASD symptoms and SAD in individuals with ASD found that SAD symptoms were associated with poorer social skills and functioning [13]. Additionally, studies have reported high rates of ADHD in adult patients with SAD [14, 15]. In particular, the presence of comorbid ADHD has been associated with increased severity and functional impairment on the Global Assessment of Functioning Scale [16]. Thus, the relationship between SAD and ADHD/ASD has been studied in terms of epidemiology and social function.

SAD was previously known as "social phobia," a term coined by Janet in the early 1900s [17]. However, the concept of SAD did not exist in the U.S. until Marks and Gelder differentiated social phobia from phobic disorders in the 1960s [18]. Meanwhile, in Japan, SAD has been reported since the 1920s. Morita initially termed the features of SAD as Taijin-Kyofu-sho (TK), which literally means disorder (sho) of fear (kyofu) of interpersonal relations (taijin) $[19,20]$. TK is defined as persistent and excessive fear of offending others in social situations with one's physical characteristics, such as blushing, gaze, or body odor [21]. It has been considered a subtype of neurosis or neurasthenic state, which is called Shinkeishitsu in Japanese [20] and is regarded as a culture-bound anxiety disorder in Japan and other East Asian countries [22]. In the DSM-5, TK is listed under SAD and briefly described in the section on culture-related diagnostic issues [11].

The psychopathologies of TK and SAD have been compared. There are two types of TK: "tension" and "offensive" [23]. In the tension type, patients fear being looked down upon in social situations because of a physical manifestation of anxiety or embarrassment, such as blushing. They also feel ashamed for experiencing these anxieties and fears and therefore avoid anxiety-provoking social situations. These features resemble the "fear of being noticed" seen in patients with SAD. Furthermore, Nakamura et al. compared the clinical diagnosis of TK and the operational diagnosis of SAD according to the DSM-IIIR. They found that $65.8 \%$ of patients with TK were also diagnosed with SAD [24], suggesting that TK and SAD share clinical and psychopathological features.

The offensive type of TK is characterized by fear of offending or disgusting others due to eye contact or body odor. Patients with offensive type TK believe that they are offending others with their physical appearance or that others are avoiding them [20]. Previously, the major difference between TK and SAD was considered to be that patients with TK fear offending others, whereas patients with SAD fear embarrassing themselves [25]. Therefore, TK has been described as an other-oriented (allocentric) disorder and SAD as a self-oriented (egocentric) disorder [26].

With the publication of the DSM-5, the differences between the features of TK and SAD have become ambiguous. The following sentence was added to the DSM-5 as "criterion B" of SAD: "The individual fears that he or she will act in a way or show anxiety symptoms that will be negatively evaluated (i.e., will be humiliating or embarrassing; will lead to rejection or offend others)" [11]. The definition of SAD was expanded because TK has been observed not only in East Asians but also in individuals from Western countries $[27,28]$. However, some subcategories of TK are difficult to classify into the DSM-5 criteria of SAD (e.g., Shubo-kyofu, the phobia of a deformed body, and Jikoshu-kyofu, the phobia of one's own foul body odor) $[25,29]$. Thus, although TK has been regarded as a type of SAD, it is still controversial whether these two disorders are identical because of several differences in their clinical features.

Since TK and SAD are unlikely to be identical in some respects, we considered it useful to focus on the differences in the causes of these two anxiety disorders. To examine the differences in psychopathology between TK and SAD, we previously compared SAD and TK in terms of ADHD traits [30]. However, our previous study did not compare TK and SAD with respect to other NDDs. Here, we hypothesized that the ADHD and ASD traits may contribute to the onset of TK and SAD. This study is aimed at examining the association of TK and SAD with the ADHD and ASD traits in university students.

\section{Materials and Methods}

2.1. Study Design and Subjects. This cross-sectional study was conducted at Kyushu University from April 2015 to April 2018. All participants were Japanese students at the Department of Interdisciplinary Graduate School of Engineering Sciences and Faculty of Arts and Science at Kyushu University who volunteered to participate in the study. We obtained informed consent from the students as follows: students were provided with our research manual and received an oral explanation of the study (purpose, methods, risk, and the right to withdraw from the study). We then asked students enrolled in psychology, health, and safety courses to volunteer. The questionnaires were completed anonymously, and the answers did not contain any identifying information. Submission of the questionnaire was regarded as consent for participation in the study. Furthermore, the participants were provided opportunities to opt-out from the study. Students who had previously been administered the scales used in the study were excluded to avoid duplication. This study was approved by the Ethics Committee of the Faculty of Arts and Science, Kyushu University, Japan (approval number: 201406R). 


\subsection{Measures}

2.2.1. The Taijin-Kyofu-sho Scale. The Taijin-Kyofu-sho scale (TKs), developed by Kleinknecht et al. in 1997, consists of 31 items related to TK symptoms that best differentiate patients with TK from controls [19]. Participants were instructed to rate all 31 items on a seven-point scale $(1=$ totally false to $7=$ exactly true), with total TKs scores ranging from 31 to 217. Based on the study by Kleinknecht et al., participants scoring 122 or higher were considered to have TK $[19,30]$.

2.2.2. The Liebowitz Social Anxiety Scale (LSAS). We used the Japanese version of the LSAS in this study [31]. The LSAS consists of 24 items that assess the extent of social interactions and performance in situations that may trigger SAD symptoms [32]. Participants rated all items on a fourpoint scale $(0=$ none to $3=$ severe $)$ based on the fear felt during specific situations (fear or anxiety section), following which they were rated again based on the avoidance of a situation (avoidance section). Total fear/anxiety and total avoidance scores ranged from 0 to 72 each; thus, the total LSAS score ranged from 0 to 144 . Based on previous reports, participants scoring 60 or higher were considered to have SAD [33-36].

2.2.3. The Adult Attention-Deficit/Hyperactivity Disorder Self-Report Scales. The ASRS version 1.1 is a self-report questionnaire designed to screen for adult ADHD [37]. It consists of 18 items rated on a five-point scale $(0=$ never to $4=$ very often). Six of the questions (part A: items 1 to 6 ) are reported to be predictive of symptoms consistent with ADHD and are often used to define adult ADHD. Each item has a minimum score allowing a "positive" classification (2 (sometimes) or 3 (often)), and the total number of "positive" items is counted. The minimum number of positive ASRS-6 part A items for the indication of adult ADHD is 4. Accordingly, we adopted a cut-off value (COV) of 4 for the definition of the ADHD trait in this study (ASRS $-6 \geq 4$ : ADHD + ; $<4$ : ADHD -).

2.2.4. The 16-Item Autism-Spectrum Quotient (AQ16). The $\mathrm{AQ}$, developed by Baron-Cohen et al. [38], is a 50 -item self-report questionnaire that measures autistic traits. The AQ uses a four-point Likert-type scale (strongly agree, slightly agree, slightly disagree, and strongly disagree). Slightly or strongly agree responses are each scored 1 point, resulting in a total score ranging from 0 to 50 . Although it is widely used to measure autism spectrum tendencies in clinical research, the 50 -item version of the AQ is too lengthy to be included in comprehensive screening for psychiatric disorders. Therefore, shorter versions of the AQ have been developed as screening tools for ASD. To examine the extent of autistic traits in participants, we used the Japanese version of the 16-item Autism-Spectrum Quotient (AQ16), which was designed to detect Asperger's syndrome [39]. According to previous research, a COV of 12 on the AQ16 (score range: 0 to 16$)$ has high sensitivity (0.80) and specificity (0.97) [39], and we therefore adopted a COV of 12 points for the definition of the ASD trait in this study (AQ16 $\geq 12$ : ASD $+;<12$ : ASD -).
2.3. Data Analysis. First, we classified the participants into $\mathrm{TK}+$ and TK- groups based on their TKs scores (TK+ met the abovementioned criteria for TK; TK- did not meet the abovementioned criteria for TK). Similarly, they were classified into SAD+/SAD-, ADHD+/ADHD-, and ASD+/ASDgroups according to the relevant definitions. Second, we compared the prevalence of TK and SAD across the four groups based on ADHD trait/ASD trait, that is, ADHDASD-, ADHD+ ASD-, ADHD- ASD+, and ADHD+ ASD + . Third, we used multiple logistic regression analysis to examine the potential contributions of ADHD trait and ASD trait to the occurrence of TK or SAD, with TK $(-=0$, $+=1)$ or $\operatorname{SAD}(-=0,+=1)$ as the dependent variable, ADHD trait $(-=0,+=1)$ and $\operatorname{ASD}$ trait $(-=0,+=1)$ as independent variables, and age (years) and sex (male $=0$, female $=1$ ) as common covariates. Finally, because some participants had comorbid TK and SAD, we attempted to characterize them based on the differences in symptoms associated with TK and SAD. For this purpose, we further classified them into "pure TK," "pure SAD," and "TK-SAD mixed," where an individual with pure TK only met the criteria for TK but not for SAD (the opposite was true for pure SAD), and an individual with TK-SAD mixed met the criteria for both TK and SAD. We then repeated logistic regression analyses with pure TK, pure SAD, and TK-SAD mixed as dependent variables. The chi-square test or Fisher's exact test was used for comparing categorical variables between groups; the Kruskal-Wallis test was used for the other variables. Data were analyzed using SPSS version 27.0 (IBM Corporation, Armonk, NY, USA). Statistical tests were two-sided, and $p$ values $<0.05$ were considered significant.

\section{Results}

In total, 818 students (males: 628, females: 190) completed all the psychological tests. The mean age (standard deviation (SD): range) of the participants was 21.4 years (2.02: 18-40 years). Figure 1 shows the frequency distributions of the TKs (a), LSAS (b), ASRS-6 (c), and AQ16 (d) scores; the corresponding COVs are shown in each graph. Of the 818 participants, $150(18.3 \%), 86(10.5 \%)$, and $47(5.7 \%)$ were classified as pure TK, pure SAD, and TK-SAD mixed, respectively. With respect to NDDs, $182(22.2 \%), 33(4.0 \%)$, and 20 (2.4\%) had ADHD trait, ASD trait, and comorbid ADHD trait and ASD trait, respectively. The mean (SD) TKs, LSAS, ASRS-6, and AQ16 scores were 89.0 (33.7), 32.9 (20.7), 2.33 (1.48), and 5.87 (2.86), respectively.

The characteristics of the participants are shown in Table 1. The participants with TK or SAD were slightly younger, were more likely to be female, and had a higher proportion of comorbid ADHD trait or ASD trait than controls.

Figure 2 shows the prevalence of TK (a) and SAD (b) across the groups based on ADHD trait/ASD trait, that is, ADHD- ASD-, ADHD+ ASD-, ADHD- ASD+, and $\mathrm{ADHD}+\mathrm{ASD}+$. The proportion (number) of participants with TK was $14.9 \%$ (93/623), 22.8\% (37/162), 61.5\% (8/13), and $60.0 \%(12 / 20)$ in the ADHD- ASD-, ADHD+ ASD-, $\mathrm{ADHD}-\mathrm{ASD}+$, and $\mathrm{ADHD}+\mathrm{ASD}+$ groups, respectively. 


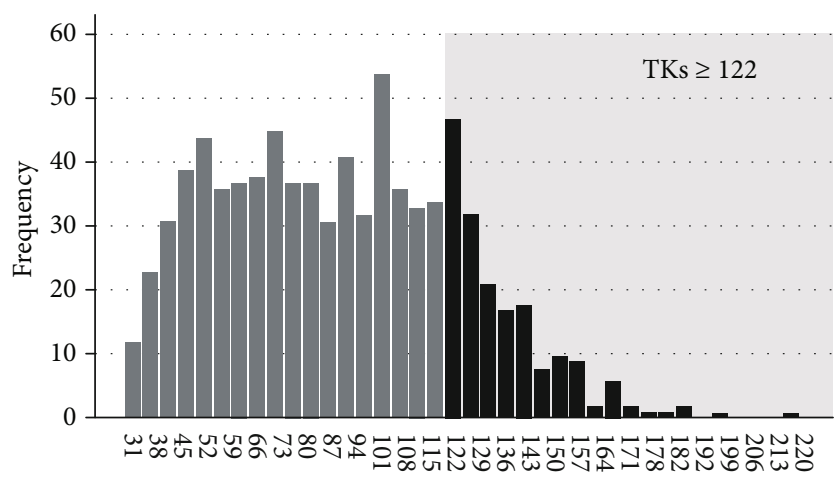

(a)

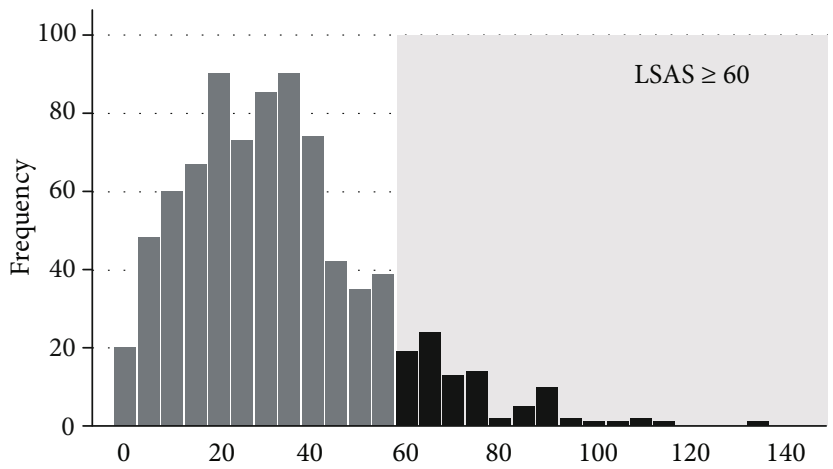

(b)

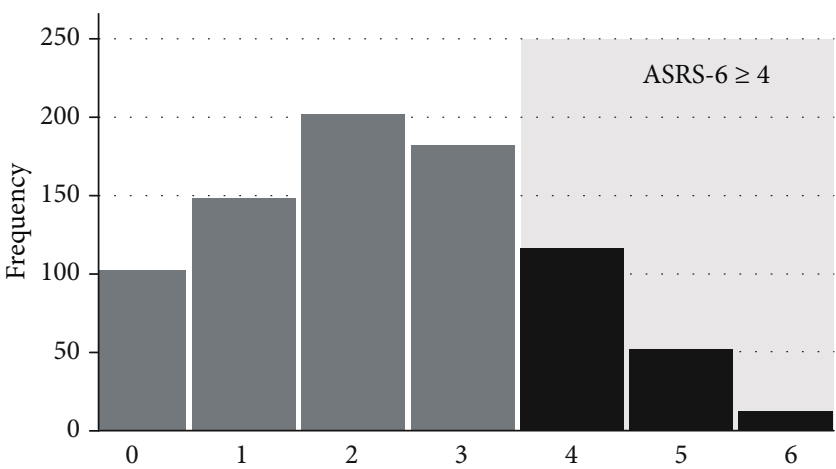

(c)

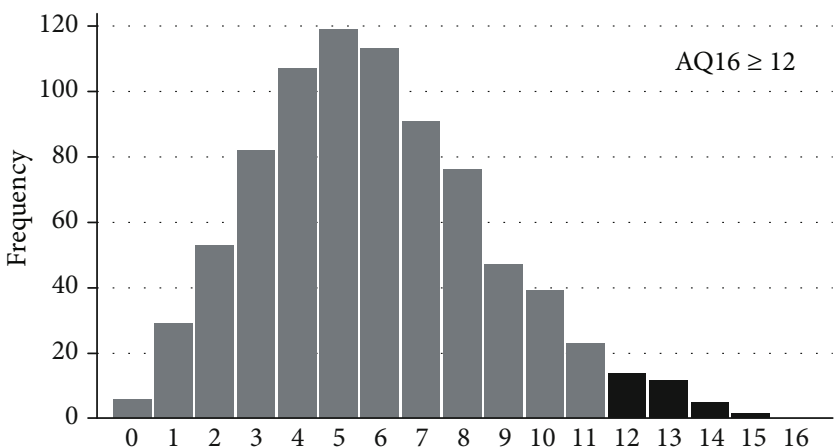

(d)

Figure 1: Histograms of the psychological test scores. The horizontal axes indicate the scores for each test. The vertical axes indicate the number of participants (frequency) with each score. The gray area indicates participants whose scores exceeded the cut-off value (COV). (a) Distribution of the Taijin-Kyofu-sho scale (TKs) scores. COV: 122. (b) Distribution of the Liebowitz Social Anxiety Scale (LSAS) scores. COV: 60. (c) Distribution of the adult Attention-Deficit/Hyperactivity Disorder Self-Report Scale-6 (ASRS-6) scores. COV: 4. (d) Distribution of the 16-item Autism-Spectrum Quotient (AQ16) scores. COV: 12. 
TABLE 1: Characteristics of participants.

\begin{tabular}{|c|c|c|c|c|}
\hline & $\begin{array}{c}\text { Control }(\mathrm{TKs}<122, \\
\text { LSAS }<60)\end{array}$ & $\begin{array}{l}\text { Participants with } \\
\text { TK (TKs } \geq 122)\end{array}$ & $\begin{array}{l}\text { Participants with } \\
\text { SAD (LSAS } \geq 60 \text { ) }\end{array}$ & $p$ value \\
\hline Number & 629 & $150 \dagger$ & $86 \dagger$ & - \\
\hline Mean age (standard deviation) & $21.4(2.01)$ & $21.1(2.01)$ & $20.7(2.16)$ & $0.008^{+}$ \\
\hline Sex, F/M (\% F) & $129 / 500(20.5 \%)$ & $44 / 106(29.3 \%)$ & $36 / 50(41.9 \%)$ & $p<0.001^{\#}$ \\
\hline $\mathrm{ADHD}$ trait $(\%)$ & $118(18.8 \%)$ & $49(32.7 \%)$ & $39(45.3 \%)$ & $p<0.001^{\#}$ \\
\hline ASD trait $(\%)$ & $11(1.7 \%)$ & $20(13.3 \%)$ & $15(17.4 \%)$ & $p<0.001^{\#}$ \\
\hline
\end{tabular}

TK: Taijin-Kyofu-sho; SAD: social anxiety disorder; TKs: Taijin-Kyofu-sho scale; LSAS: Liebowitz Social Anxiety Scale; M: male; F: female; ADHD: attentiondeficit/hyperactivity disorder; ASD: autism spectrum disorder. $†$ There were 47 cases of comorbid TK and SAD. ${ }^{+} p<0.01$, Kruskal-Wallis test; ${ }^{*} p<0.001$, chisquare test.

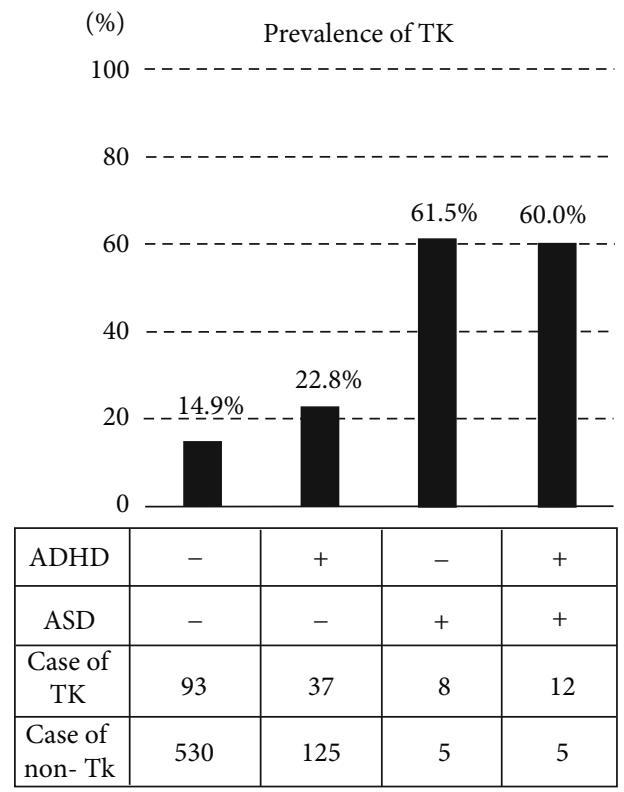

(a)

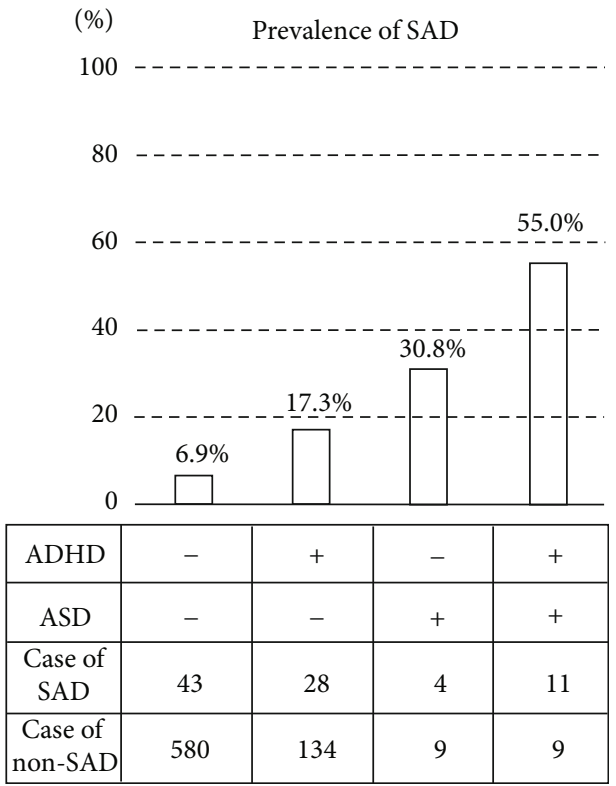

(b)

Figure 2: Prevalence of Taijin-Kyofu-sho and social anxiety disorder in participants with neurodevelopmental disorder traits. Participants were divided into four groups (ADHD - ASD -, ADHD + ASD -, ADHD - ASD +, and ADHD + ASD +) to examine the groupwise prevalence of TK and SAD. (a) Black bars indicate the prevalence of TK. (b) White bars indicate the prevalence of SAD. ADHD: attention-deficit/hyperactivity disorder; ASD: autism spectrum disorder; TKs: Taijin-Kyofu-sho; SAD: social anxiety disorder.

The proportion (number) of participants with SAD was $6.9 \%$ (43/623), $17.3 \%$ (28/162), 30.8\% (4/13), and $55.0 \%$ $(11 / 20)$ in the ADHD- ASD-, ADHD+ ASD-, ADHD$\mathrm{ASD}+$, and $\mathrm{ADHD}+\mathrm{ASD}+$ groups, respectively. Thus, the prevalence of TK/SAD differed based on the presence/absence of ADHD trait/ASD trait, and the pattern of association with ADHD trait/SD trait seemed to be substantially different between TK and SAD. Indeed, there was a statistically significant difference in the prevalence of TK and SAD among the groups ((a) $p<0.001$, (b) $p<0.001$, Fisher's exact test).

As shown in Figure 2, the presence of ADHD trait/ASD trait resulted in differences in the proportions of $\mathrm{TK}$ and SAD. To confirm whether ADHD trait and ASD trait were associated with $\mathrm{TK}$ and $\mathrm{SAD}$, logistic regression analyses were conducted with TK or SAD $(0=$ control, $1=\mathrm{TK}$ or $\mathrm{SAD})$ as a binary outcome and age, sex, ADHD trait, and ASD trait as variables. As shown in Table 2, among the four variables, ADHD trait and ASD trait were significantly asso- ciated with TK (ADHD trait: $p=0.032$, odds ratio (OR) 1.57; ASD trait: $p<0.001$, OR 6.63). Female sex, ADHD trait, and ASD trait were significantly associated with SAD (female sex: $p=0.003$, OR 2.29; ADHD trait: $p<0.001$, OR 2.72; ASD trait: $p<0.001$, OR 6.46).

We found that ADHD trait and ASD trait were both significantly associated with TK and SAD (Table 2). However, these analyses included 47 cases of comorbid TK and SAD. The characteristics of the participants grouped according to the presence or absence of TK and SAD are shown in Table 3. ASD was significantly associated with pure TK (ASD: $p=0.006$; OR 3.99). Female sex and ADHD trait were significantly associated with pure $\mathrm{SAD}$ (sex (female): $p=$ 0.013, OR 2.61; ADHD trait: $p=0.012$, OR 2.46). Female sex, ADHD trait, and ASD trait were significantly associated with TK-SAD mixed (sex (female): $p=0.043$, OR 2.16; ADHD trait: $p=0.003$, OR 2.75; ASD trait: $p<0.001$, OR 16.93) (Table 4). 
TABLE 2: Factors associated with TK and SAD on logistic regression analysis.

\begin{tabular}{lcccc}
\hline Factor & OR $(95 \%$ CI $)$ & TK & OR (95\% CI) & SAD \\
\hline Age & $0.97(0.87-1.07)$ & 0.48 & $0.90(0.79-1.03)$ & 0.12 \\
Female sex & $1.37(0.87-2.15)$ & 0.17 & $2.29(1.33-3.96)$ & 0.003 \\
ADHD trait & $1.58(1.04-2.39)$ & 0.032 & $2.72(1.66-4.46)$ & $<0.001$ \\
ASD trait & $6.63(3.15-13.98)$ & $<0.001$ & $6.46(2.93-14.25)$ & $<0.001$ \\
\hline
\end{tabular}

Italic text indicates statistical significance. TK: Taijin-Kyofu-sho; SAD: social anxiety disorder; OR: odds ratio; CI: confidence interval; ADHD: attentiondeficit/hyperactivity disorder; ASD: autism spectrum disorder.

TABLE 3: Characteristics of participants classified according to TKs and LSAS cut-off values.

\begin{tabular}{|c|c|c|c|c|c|}
\hline & $\begin{array}{c}\text { Control LSAS } \\
(<60) / \mathrm{TKs}(<122)\end{array}$ & $\begin{array}{c}\text { Pure TK LSAS } \\
(<60) / \mathrm{TKs}(\geq 122)\end{array}$ & $\begin{array}{l}\text { Pure SAD LSAS } \\
(\geq 60) / \mathrm{TKs}(<122)\end{array}$ & $\begin{array}{l}\text { TK-SAD mixed LSAS } \\
(\geq 60) / \mathrm{TKs}(\geq 122)\end{array}$ & $p$ value \\
\hline Number & 629 & 103 & 39 & 47 & \\
\hline Mean age (SD) & $21.4(2.01)$ & $21.3(1.81)$ & $20.7(1.92)$ & $20.7(2.37)$ & $0.03^{\mathrm{a} *}$ \\
\hline Sex, F/M (\% F) & $129 / 500(20.5 \%)$ & $25 / 78(24.3 \%)$ & $17 / 22(43.6 \%)$ & $19 / 28(40.4 \%)$ & $<0.001^{\mathrm{b} * *}$ \\
\hline Mean ASRS-6 score (SD) & $2.19(1.45)$ & $2.52(1.36)$ & $2.74(1.43)$ & $3.45(1.56)$ & $<0.001^{\mathrm{a} * *}$ \\
\hline Mean AQ16 score (SD) & $5.37(2.60)$ & $7.09(2.83)$ & $6.85(2.59)$ & $9.40(2.92)$ & $<0.001^{\mathrm{a} * *}$ \\
\hline
\end{tabular}

TK: Taijin-Kyofu-sho; SAD: social anxiety disorder; LSAS: Liebowitz Social Anxiety Scale; TKs: Taijin-Kyofu-sho scale; M: male; F: female; SD: standard deviation; ASRS-6: Attention-Deficit/Hyperactivity Disorder Self-Report Scale-6; AQ16: 16-item Autism-Spectrum Quotient (AQ16). ${ }^{a}$ Kruskal-Wallis test; b chi-square test; ${ }^{*} p<0.05,{ }^{* *} p<0.001$.

TABLE 4: Factors associated with pure TK, pure SAD, and TK-SAD mixed on logistic regression analysis.

\begin{tabular}{|c|c|c|c|c|c|c|}
\hline & \multicolumn{2}{|c|}{ Pure TK } & \multicolumn{2}{|c|}{ Pure SAD } & \multicolumn{2}{|c|}{ TK-SAD mixed } \\
\hline & OR (95\% CI) & $p$ value & OR $(95 \% \mathrm{CI})$ & $p$ value & OR (95\% CI) & $p$ value \\
\hline Age & $0.98(0.87-1.10)$ & 0.73 & $0.91(0.75-1.09)$ & 0.309 & $0.86(0.72-1.03)$ & 0.11 \\
\hline Female sex & $1.24(0.73-2.11)$ & 0.43 & $2.61(1.23-5.55)$ & 0.013 & $2.16(1.03-4.56)$ & 0.043 \\
\hline ADHD trait & $1.26(0.76-2.09)$ & 0.38 & $2.46(1.22-4.97)$ & 0.012 & $2.75(1.40-5.42)$ & 0.003 \\
\hline ASD trait & $3.99(1.47-10.78)$ & 0.006 & $3.82(0.54-14.68)$ & 0.22 & $16.93(6.37-45.01)$ & $<0.001$ \\
\hline
\end{tabular}

Italic text indicates statistical significance. TK: Taijin-Kyofu-sho; SAD: social anxiety disorder; OR: odds ratio; CI: confidence interval; ADHD: attentiondeficit/hyperactivity disorder; ASD: autism spectrum disorder.

\section{Discussion}

In this study, we found that a higher proportion of participants with TK and SAD had ADHD trait and ASD trait than those in the control group. Using logistic regression analysis, we identified ADHD trait and ASD trait as factors associated with TK. In addition to ADHD trait and ASD trait, female sex was significantly associated with SAD. Furthermore, to highlight the differences between the clinical features of $\mathrm{TK}$ and $\mathrm{SAD}$, we divided the participants into four groups (control, pure TK, pure SAD, and TK-SAD mixed) and performed logistic regression analyses. ASD trait was associated with pure TK, and female sex and ADHD trait were associated with pure SAD. We believe this is the first study comparing TK and SAD according to NDDs (ADHD and ASD). Our results may provide new insights into the pathogenesis of TK and SAD, which can contribute to the early management of these anxiety disorders.

Although few studies have examined the prevalence of TK among patients with developmental disorders, many have shown a relationship between SAD and NDDs in adults. Studies from the U.S. and Norway have reported that
29.3\% [40] and 14.2\% [41] of adults with ADHD had comorbid SAD, respectively. Here, $21.4 \%$ (39/182) of participants with ADHD trait had SAD, which was in line with the results of previous reports.

Adults with ASD have been reported to have a significantly higher prevalence of SAD than those without ASD (21.7\% vs. 5.0\%) [42]. Furthermore, a systematic review estimated the prevalence of SAD in adults with ASD as $29 \%$ [43]. However, we observed a higher prevalence of SAD in participants with ASD trait $(45.5 \%(15 / 33))$ than that in previous reports. This may be explained by differences in the participants' backgrounds or the evaluation method used. Our research focused on university students, whereas other studies included participants with various backgrounds (e.g., education, occupation, economic status, and race). Moreover, participants of previous studies were clinically diagnosed with ASD based on the DSM or International Classification of Diseases criteria, in contrast to the selfadministered test (AQ16) used in our study.

Logistic regression analysis identified $\mathrm{ADHD}$ trait and ASD trait as common factors associated with TK and SAD. However, the analysis included 47 cases of comorbid TK 
and SAD. To exclude the effect of TK and SAD on each other, we divided the participants into pure TK and pure SAD groups and performed logistic regression analysis. As a result, ASD trait was identified as a factor associated with pure TK, and ADHD trait and female sex were identified as factors associated with pure SAD.

One possible reason for the association between ASD trait and TK is collectivism. Contrary to individualism in Western countries, East Asian societies, including Japan, are characterized by collectivism. In collectivism, people need to sensitively perceive the feelings or thoughts of others because "not bothering others" and "cooperativeness" are regarded as virtues; that is, the fear of being disliked and not accepted plays a prominent role in collectivistic societies like Japan [44]. However, patients with ASD have deficits in empathy and "theory of mind," and they often have difficulties in understanding the thoughts of others and communicating with them properly $[45,46]$. Therefore, patients with ASD are unable to form and maintain positive peer relationships, and some of them are even bullied in childhood. Indeed, there is a growing body of literature indicating that patients with ASD are at a significantly higher risk of both victimization and perpetration [47]. It is possible that these interpersonal failures and painful experiences may make patients with ASD oversensitive to communication with others; that is, some patients with ASD may always worry that their words and actions may hurt others' feelings or make them uncomfortable. This anxiety about "making peers uncomfortable" is a core feature of TK.

Another reason for the association between ASD trait and TK may be social withdrawal. Social withdrawal, a maladaptive behavior associated with ASD, is more prevalent among children with ASD than typically developing children and often worsens with age [48]. Meanwhile, patients with severe TK are often socially withdrawn. This state is called Hikikomori, which literally means "pulling inward" in Japanese. The definition of Hikikomori is abnormal avoidance of social contact, typically by adolescents [49]. Indeed, a case series of inpatients with TK revealed that $30 \%$ of them could be regarded as Hikikomori [50]. Moreover, using the AQ, Katsuki et al. have shown that patients with Hikikomori were more likely to have autistic tendencies than non-Hikikomori individuals [51]. Thus, social withdrawal in TK and Hikikomori, which was first described in Japan, seems to be closely related to ASD.

Accumulating evidence suggests that SAD is closely associated with ADHD. Koyuncu et al. reported that the rate of childhood ADHD in SAD was high (72.3\%) [16]. In a cross-sectional observational study, Sanz et al. found that adolescents with inattentive ADHD had a higher degree of SAD [52]. Furthermore, Nelson and Liebel demonstrated that college students with ADHD have significantly more anxiety symptoms than those without ADHD [53]. In their report, participants with inattentive ADHD had more anxiety symptoms than in those with combined ADHD (inattentive and hyperactive-impulsive ADHD). Thus, considering the close relationship between SAD and $\mathrm{ADHD}$, it is not surprising that $\mathrm{ADHD}$ trait is associated with pure SAD.
Previous studies have consistently shown that women are more likely to have SAD, to have more severe clinical symptoms of SAD, and to have greater subjective distress compared to men $[54,55]$. Here, consistent with their results, we found that female sex was associated with pure SAD. However, sex differences in TK remain controversial. TK reportedly has a male predominance of approximately $3: 2$ [17]. However, Maeda and Nathan reported that the proportion of female patients with TK was increasing [20]. Furthermore, Essau et al. compared British and Japanese young adults using the TKs and found that British females had significantly higher scores than males; however, no significant sex differences were found in a previous analysis of the Japanese population [56]. We found no sex differences in pure TK on logistic regression analysis. Epidemiological studies with larger samples are needed to examine the sex differences in TK.

4.1. Limitations of This Study. This is the first study to examine the association between two types of anxiety disorders (TK and SAD) and developmental disorders (ADHD and ASD) using logistic regression analysis. However, it has some limitations. First, all the participants were recruited from a single university, and $70 \%$ of the students were male. Moreover, Kyushu University is ranked as one of the top 10 universities in Japan, due to which the intelligence level of its students is expected to be relatively high. Therefore, our results cannot be generalized to all Japanese young adults. Second, we relied on self-reported assessments to diagnose $\mathrm{TK}, \mathrm{SAD}, \mathrm{ADHD}$, and ASD. In general, a clinical diagnosis should be made according to the operational diagnostic criteria based on a psychiatric interview. Therefore, a clinical evaluation using structured interviews is required for the accurate diagnosis of these disorders. We also emphasize that our study investigated the traits of each disorder and did not intend to diagnose any of these disorders. Moreover, the TK was developed to evaluate the extent of TK symptoms, and further research is required to ascertain whether it is suitable for diagnosing TK.

\section{Conclusions}

In this study, we examined the association between anxiety disorders (TK and SAD) and the ADHD and ASD traits. Multivariate logistic regression analyses revealed that ASD was significantly and independently associated with TK, and ADHD trait and female sex were significantly and independently associated with SAD. Differences in ADHD and ASD traits may influence the future onset of TK or SAD in Japanese adults.

\section{Data Availability}

The data used in this study are available from the corresponding author upon request.

\section{Disclosure}

This research has been posted as a preprint at Research Square [57]. 


\section{Conflicts of Interest}

The authors declare that there is no conflict of interest regarding the publication of this article.

\section{Acknowledgments}

We would like to thank all of the staff at Kyushu University for recruiting the participants. We would like to thank Editage (http://www.editage.jp) for English language editing.

\section{References}

[1] American Psychiatric Association, "Neurodevelopmental disorders," in Diagnostic and Statistical Manual of Mental Disorders (DSM-5), pp. 31-86, American Psychiatric Association, Washington, DC, USA, 5th edition, 2013.

[2] T. W. Frazier, E. A. Youngstrom, J. J. Glutting, and M. W. Watkins, "ADHD and achievement: meta-analysis of the child, adolescent, and adult literatures and a concomitant study with college students," Journal of Learning Disabilities, vol. 40, no. 1, pp. 49-65, 2007.

[3] K. R. Murphy, R. A. Barkley, and T. Bush, "Young adults with attention deficit hyperactivity disorder: subtype differences in comorbidity, educational, and clinical history," The Journal of Nervous and Mental Disease, vol. 190, no. 3, pp. 147-157, 2002.

[4] J. D. McLeod, E. Meanwell, and A. Hawbaker, "The experiences of college students on the autism spectrum: a comparison to their neurotypical peers," Journal of Autism and Developmental Disorders, vol. 49, no. 6, pp. 2320-2336, 2019.

[5] T. E. Rosen, C. A. Mazefsky, R. A. Vasa, and M. D. Lerner, "Co-occurring psychiatric conditions in autism spectrum disorder," International Review of Psychiatry, vol. 30, no. 1, pp. 40-61, 2018.

[6] M. G. Mosner, J. L. Kinard, J. S. Shah et al., "Rates of cooccurring psychiatric disorders in autism spectrum disorder using the Mini International Neuropsychiatric Interview," Journal of Autism and Developmental Disorders, vol. 49, no. 9, pp. 3819-3832, 2019.

[7] A. D. Anastopoulos, G. J. DuPaul, L. L. Weyandt et al., "Rates and patterns of comorbidity among first-year college students with ADHD," J Clin Child Adolesc Psychol, vol. 47, no. 2, pp. 236-247, 2018.

[8] G. Zukerman, G. Yahav, and E. Ben-Itzchak, "Increased psychiatric symptoms in university students with autism spectrum disorder are associated with reduced adaptive behavior," Psychiatry Research, vol. 273, pp. 732-738, 2019.

[9] D. Mason, H. McConachie, D. Garland, A. Petrou, J. Rodgers, and J. R. Parr, "Predictors of quality of life for autistic adults," Autism Research, vol. 11, no. 8, pp. 1138-1147, 2018.

[10] S. P. Cuffe, S. N. Visser, J. R. Holbrook et al., "ADHD and psychiatric comorbidity: functional outcomes in a school-based sample of children," Journal of Attention Disorders, vol. 24, no. 9, pp. 1345-1354, 2020.

[11] American Psychiatric Association, "Social anxiety disorder (social phobia)," in Diagnostic and Statistical Manual of Mental Disorders (DSM-5), pp. 202-208, American Psychiatric Association, Washington, DC, USA, 5th edition, 2013.

[12] M. Freeth, T. Bullock, and E. Milne, "The distribution of and relationship between autistic traits and social anxiety in a UK student population," Autism, vol. 17, no. 5, pp. 571-581, 2013.
[13] D. Spain, J. Sin, K. B. Linder, J. McMahon, and F. Happé, "Social anxiety in autism spectrum disorder: a systematic review," Research in Autism Spectrum Disorders, vol. 52, pp. 51-68, 2018.

[14] A. Koyuncu, F. Celebi, E. Ertekin, B. E. Kok, and R. Tukel, "Attention deficit and hyperactivity in social anxiety disorder: relationship with trauma history and impulsivity," $A D H D$ Attention Deficit and Hyperactivity Disorders, vol. 8, no. 2, pp. 95-100, 2016.

[15] M. Van Ameringen, C. Mancini, W. Simpson, and B. Patterson, "Adult attention deficit hyperactivity disorder in an anxiety disorders population," CNS Neuroscience \& Therapeutics, vol. 17, no. 4, pp. 221-226, 2011.

[16] A. Koyuncu, E. Ertekin, C. Yuksel et al., "Predominantly inattentive type of ADHD is associated with social anxiety disorder," Journal of Attention Disorders, vol. 19, no. 10, pp. 856864, 2015.

[17] T. Takahashi, "Social phobia syndrome in Japan," Comprehensive Psychiatry, vol. 30, no. 1, pp. 45-52, 1989.

[18] I. M. Marks and M. G. Gelder, "Different ages of onset in varieties of phobia," American Journal of Psychiatry, vol. 123, no. 2, pp. 218-221, 1966.

[19] R. A. Kleinknecht, D. L. Dinnel, E. E. Kleinknecht, N. Hiruma, and N. Harada, "Cultural factors in social anxiety: A comparison of social phobia symptoms and Taijin Kyofusho," Journal of Anxiety Disorders, vol. 11, no. 2, pp. 157-177, 1997.

[20] F. Maeda and J. H. Nathan, "Editorial," Journal of Psychosomatic Research, vol. 46, no. 6, pp. 525-530, 1999.

[21] N. Vriends, M. C. Pfaltz, P. Novianti, and J. Hadiyono, "Taijin Kyofusho and social anxiety and their clinical relevance in Indonesia and Switzerland," Frontiers in Psychology, vol. 4, no. 3, 2013.

[22] S. G. Hofmann and D. E. Hinton, "Cross-cultural aspects of anxiety disorders," Current Psychiatry Reports, vol. 16, no. 6, p. 450, 2014.

[23] T. Nagata, F. Suzuki, and A. R. Teo, "Generalized social anxiety disorder: a still-neglected anxiety disorder 3 decades since Liebowitz's review," Psychiatry and Clinical Neurosciences, vol. 69, no. 12, pp. 724-740, 2015.

[24] K. Nakamura, K. Kitanishi, Y. Miyake, K. Hashimoto, and M. Kubota, "The neurotic versus delusional subtype of Taijin-Kyofu-sho: their DSM diagnoses," Psychiatry and Clinical Neurosciences, vol. 56, no. 6, pp. 595-601, 2002.

[25] L. Lim, "Taijin-Kyofu-Sho: a subtype of social anxiety," Open Journal of Psychiatry, vol. 3, no. 4, pp. 393-398, 2013.

[26] S. C. Chang, "Social anxiety (phobia) and East Asian culture," Depression and Anxiety, vol. 5, no. 3, pp. 115-120, 1997.

[27] Y. Choy, F. R. Schneier, R. G. Heimberg, K. S. Oh, and M. R. Liebowitz, "Features of the offensive subtype of Taijin-KyofuSho in US and Korean patients with DSM-IV social anxiety disorder," Depression and Anxiety, vol. 25, no. 3, pp. 230240, 2008.

[28] J. Kim, R. M. Rapee, and J. E. Gaston, "Symptoms of offensive type Taijin-Kyofusho among Australian social phobics," Depression and Anxiety, vol. 25, no. 7, pp. 601-608, 2008.

[29] K. Suzuki, N. Takei, M. Kawai, Y. Minabe, and N. Mori, "IsTaijin Kyofushoa culture-bound syndrome?," American Journal of Psychiatry, vol. 160, no. 7, p. 1358, 2003.

[30] K. Kajitani, R. Tsuchimoto, T. Matsushita, and H. Fukumori, "Correlation between Taijin-Kyofu-sho and attention deficit hyperactivity disorder among university students: a self- 
reported assessment study," American Journal of Psychiatry, vol. 2019, article 7953123, pp. 1-8, 2019.

[31] S. Asakura, S. Inoue, F. Sasaki et al., "Reliability and validity of the Japanese version of the Liebowitz Social Anxiety Scale," Seishin Igaku, vol. 44, pp. 1077-1084, 2002.

[32] M. R. Liebowitz, J. M. Gorman, A. J. Fyer, and D. F. Klein, "Social phobia. Review of a neglected anxiety disorder," Archives of General Psychiatry, vol. 42, no. 7, pp. 729-736, 1985.

[33] S. Sasai, S. Asakura, T. Koyama, T. Hayano, and A. Hagino, "Long-term administration of escitalopram in patients with social anxiety disorder in Japan," Neuropsychiatric Disease and Treatment, vol. 12, pp. 1817-1825, 2016.

[34] M. B. Stein, L. N. Ravindran, N. M. Simon et al., "Levetiracetam in generalized social anxiety Disorder," The Journal of Clinical Psychiatry, vol. 71, no. 5, pp. 627-631, 2010.

[35] N. K. Rytwinski, D. M. Fresco, R. G. Heimberg et al., "Screening for social anxiety disorder with the self-report version of the Liebowitz Social Anxiety Scale," Depression and Anxiety, vol. 26, no. 1, pp. 34-38, 2009.

[36] T. Zaitsu, M. Ueno, K. Shinada, F. A. Wright, and Y. Kawaguchi, "Social anxiety disorder in genuine halitosis patients," Health Qual Life Outcomes, vol. 9, no. 1, p. 94, 2011.

[37] R. C. Kessler, L. Adler, M. Ames et al., "The World Health Organization Adult ADHD Self-Report Scale (ASRS): a short screening scale for use in the general population," Psychological Medicine, vol. 35, no. 2, pp. 245-256, 2005.

[38] S. Baron-Cohen, S. Wheelwright, R. Skinner, J. Martin, and E. Clubley, "The autism-spectrum quotient (AQ): evidence from Asperger syndrome/high-functioning autism, males and females, scientists and mathematicians," Journal of Autism and Developmental Disorders, vol. 31, no. 1, pp. 5-17, 2001.

[39] H. Kurita, H. Osada, Y. Miyamoto, and K. Shimizu, "AutismSpectrum Quotient Japanese Version (AQ-J) cutoff for Asperger's disorder," Jpn J Clin Psychiatry, vol. 33, pp. 209-214, 2004.

[40] R. C. Kessler, L. Adler, R. Barkley et al., "The prevalence and correlates of adult ADHD in the United States: results from the National Comorbidity Survey Replication," American Journal of Psychiatry, vol. 163, no. 4, pp. 716-723, 2006.

[41] E. Anker, B. Bendiksen, and T. Heir, "Comorbid psychiatric disorders in a clinical sample of adults with ADHD, and associations with education, work and social characteristics: a cross-sectional study," BMJ Open, vol. 8, no. 3, article e019700, 2018.

[42] A. G. Lever and H. M. Geurts, "Psychiatric co-occurring symptoms and disorders in young, middle-aged, and older adults with autism spectrum disorder," Journal of Autism and Developmental Disorders, vol. 46, no. 6, pp. 1916-1930, 2016.

[43] M. J. Hollocks, J. W. Lerh, I. Magiati, R. Meiser-Stedman, and T. S. Brugha, "Anxiety and depression in adults with autism spectrum disorder: a systematic review and meta-analysis," Psychological Medicine, vol. 49, no. 4, pp. 559-572, 2019.

[44] H. Hashimoto and T. Yamagishi, "Two faces of interdependence: harmony seeking and rejection avoidance," Asian Journal of Social Psychology, vol. 16, no. 2, pp. 142-151, 2013.

[45] I. E. Harmsen, "Empathy in autism spectrum disorder," Journal of Autism and Developmental Disorders, vol. 49, no. 10, pp. 3939-3955, 2019.

[46] S. Baron-Cohen, A. M. Leslie, and U. Frith, "Does the autistic child have a "theory of mind" ?," Cognition, vol. 21, no. 1, pp. 37-46, 1985.
[47] J. H. Schroeder, M. C. Cappadocia, J. M. Bebko, D. J. Pepler, and J. A. Weiss, "Shedding light on a pervasive problem: a review of research on bullying experiences among children with autism spectrum disorders," Journal of Autism and Developmental Disorders, vol. 44, no. 7, pp. 1520-1534, 2014.

[48] D. K. Anderson, M. P. Maye, and C. Lord, "Changes in maladaptive behaviors from midchildhood to young adulthood in autism spectrum disorder," American Journal on Intellectual and Developmental Disabilities, vol. 116, no. 5, pp. 381-397, 2011.

[49] T. A. Kato, S. Kanba, and A. R. Teo, "Hikikomori: multidimensional understanding, assessment, and future international perspectives," Psychiatry and Clinical Neurosciences, vol. 73, pp. 427-440, 2019.

[50] K. Nakamura and R. Shioji, "Taijin Kyoufushou to Hikikomori," Rinshou Seishin Igaku, vol. 26, pp. 1169-1176, 1997.

[51] R. Katsuki, A. Inoue, S. Indias et al., "Clarifying deeper psychological characteristics of Hikikomori using the Rorschach comprehensive system: a pilot case-control study," Frontiers in Psychiatry, vol. 10, p. 412, 2019.

[52] M. J. Mardomingo Sanz, C. Sancho Mateo, B. Soler López et al., "Assessment of comorbidity and social anxiety in adolescents with attention deficit hyperactivity disorder: the SELFIE study," Anales de Pediatría, vol. 90, no. 6, pp. 349-361, 2019.

[53] J. M. Nelson and S. W. Liebel, "Anxiety and depression among college students with attention-deficit/hyperactivity disorder (ADHD): cross-informant, sex, and subtype differences," Journal of American College Health, vol. 66, no. 2, pp. 123-132, 2018.

[54] M. Asher and I. M. Aderka, "Gender differences in social anxiety disorder," Journal of Clinical Psychology, vol. 74, no. 10, pp. 1730-1741, 2018.

[55] D. J. Stein, L. CCW, A. M. Roest et al., "The cross-national epidemiology of social anxiety disorder: data from the World Mental Health Survey Initiative," BMC Medicine, vol. 15, no. 1, p. 143, 2017.

[56] C. A. Essau, S. Sasagawa, J. Chen, and Y. Sakano, "Taijin Kyofusho and social phobia symptoms in young adults in England and in Japan," Journal of Cross-Cultural Psychology, vol. 43, no. 2, pp. 219-232, 2012.

[57] K. Kajitani, R. Tsuchimoto, Y. Omodaka et al., "Neurodevelopmental disorder traits in Taijin-Kyofu-sho and social anxiety disorder: a cross-sectional study among university students," Research Square, 2021. 